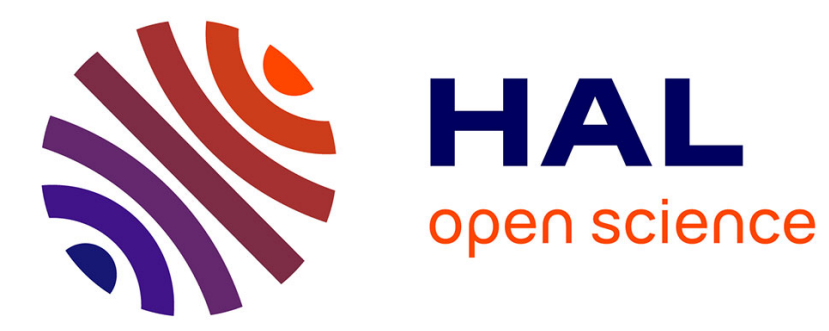

\title{
A note on improvement of adaptive observer robustness
}

\author{
Denis Efimov, Christopher Edwards, Ali Zolghadri
}

\section{To cite this version:}

Denis Efimov, Christopher Edwards, Ali Zolghadri. A note on improvement of adaptive observer robustness. 19th IFAC World Congress, Aug 2014, Cape Town, South Africa. hal-00967893

\section{HAL Id: hal-00967893 \\ https://hal.inria.fr/hal-00967893}

Submitted on 31 Mar 2014

HAL is a multi-disciplinary open access archive for the deposit and dissemination of scientific research documents, whether they are published or not. The documents may come from teaching and research institutions in France or abroad, or from public or private research centers.
L'archive ouverte pluridisciplinaire HAL, est destinée au dépôt et à la diffusion de documents scientifiques de niveau recherche, publiés ou non, émanant des établissements d'enseignement et de recherche français ou étrangers, des laboratoires publics ou privés. 


\title{
A note on improvement of adaptive observer robustness
}

\author{
Denis Efimov ${ }^{*, * * *}$ Christopher Edwards ${ }^{* *}$ Ali Zolghadri ${ }^{* * *}$ \\ * Non-A team at Inria Lille, Parc Scientifique de la Haute Borne, 40 \\ avenue Halley, 59650 Villeneuve d'Ascq, France (e-mail: \\ Denis.Efimov@inria.fr) \\ ** College of Engineering Mathematics and Physical Sciences, \\ University of Exeter, Exeter EX4 4QF, UK (e-mail: \\ C.Edwards@exeter.ac.uk) \\ *** University of Bordeaux, IMS-lab, Automatic control group, 351 \\ cours de la libération, 33405 Talence, France (e-mail: \\ Ali.Zolghadri@ims-bordeaux.fr) \\ **** Department of Control Systems and Informatics, National \\ Research University ITMO, 49 Kronverkskiy av., Saint Petersburg, \\ 197101, Russia
}

\begin{abstract}
In this paper the problem of adaptive observer design in the presence of disturbances is studied, and an augmented adaptive observer is proposed. First, the $H_{\infty}$ gain of a conventional adaptive observer is estimated, which characterizes the effect of disturbances on output errors. Next, it is shown that if the disturbance is "matched" in the plant equations, then it is possible to introduce additional sliding-mode feedback, dependent on the plant output, improving the accuracy of observation. Simulation results confirm the improvement.
\end{abstract}

\section{INTRODUCTION}

The problem of adaptive observer design for nonlinear systems is very challenging Besanon [2007]. In this situation the observer has to generate estimates of the vector of unknown parameters and unmeasured state components. Almost all solutions are based on special canonical representations of the uncertain nonlinear systems, that allows the observer to be designed. An important obstacle is the relative degree between the output signal and the vector of unknown parameters (i.e. the number of derivatives of the output required, before the direct dependence on the vector of unknown parameters is obtained). The observers designed in the case when the degree is one Fradkov et al. [2000] and for the higher relative degree case Fradkov et al. [2002], Xu and Zhang [2004], Zhang [2002], Efimov [2004] have completely different structures, and the dimension of the observers in the latter case is much higher.

The structure of the observer proposed in Zhang [2002] for the high relative degree case is quite sophisticated, and strongly related to a canonical form of the plant equations. A small modification to the plant structure may render the observer design impossible. To overcome this limitation, a deviation of equations of the real system from the canonical form can be modeled by external disturbances. These disturbances have to be bounded, but in some situations it is hard to assume that the disturbance is sufficiently small. Thus it is necessary to ensure robustness of the designed observers with respect to such a disturbance.

\footnotetext{
* This work was supported in part by the Government of Russian Federation (Grant 074-U01) and the Ministry of Education and Science of Russian Federation (Project 14.Z50.31.0031).
}

There exist a number of solutions aimed at improving the robustness in nonlinear systems by applying dynamic or static feedback. Some very promising solutions have been obtained in the area of sliding mode theory since sliding mode feedback is able to fully compensate for matched disturbances even granting the closed loop system finitetime stability Shtessel et al. [2013]. Recently the sliding mode approach has been successfully applied to adaptive observer design for the case of relative degree one Yan and Edwards [2008], but the application of this theory for adaptive observer design with a high relative degree is somewhat complicated due to the fixed observer structure. In this paper a method is presented for modifying the adaptive observer from Zhang [2002] using a sliding-mode feedback in the spirit of Yan and Edwards [2008], that allows us to improve the level of observer robustness with respect to some matched disturbances.

The paper is structured as follows. The adaptive observer equations and the problem statement are given in Section 2. The $H_{\infty}$ gain of the adaptive observer from Zhang [2002] is computed in Section 3. Then the sliding-mode feedback methodology is applied in Section 4. An example of a computer simulation (for a nonlinear pendulum) is presented in Section 5 to demonstrate the efficacy of the approach.

\section{PROBLEM STATEMENT}

Consider the following uncertain nonlinear system:

$$
\dot{x}=A x+\phi(y, u)+G(y, u) \theta+B v, y=C x,
$$

where $x \in \mathbb{R}^{n}, y \in \mathbb{R}^{p}, u \in \mathbb{R}^{m}$ are the state, the output and the control respectively, $\theta \in \mathbb{R}^{q}$ is the vector 
of unknown parameters, $v \in \mathbb{R}^{s}$ is the vector of external disturbances and $v: \mathbb{R}_{+} \rightarrow \mathbb{R}^{s}$ is a (Lebesgue) measurable function of time; the matrices $A, B, C$ are known and have corresponding dimensions, the functions $\phi: \mathbb{R}^{p+m} \rightarrow \mathbb{R}^{n}$ and $G: \mathbb{R}^{p+m} \rightarrow \mathbb{R}^{n \times q}$ are also assumed to be known and ensure uniqueness and existence of the system (1) solutions at least locally.

The symbol $|x|$ denotes the Euclidean norm of a vector $x$ (for a matrix $A$ the symbol $|A|$ denotes the induced matrix norm), and for the (Lebesgue) measurable functions $v$ : $\mathbb{R}_{+} \rightarrow \mathbb{R}^{s}$, the norm is defined as $\|v\|=\operatorname{ess~sup~}_{t \geq 0}\{|v(t)|\}$. For a matrix function $A: \mathbb{R}_{+} \rightarrow \mathbb{R}^{s \times q}$ we denote $\|A\|=\||A(t)|\|$. The identity matrix of dimension $n \times n$ is denoted as $I_{n}$. The symbols $\lambda_{\min }(A), \lambda_{\max }(A)$ represent the minimal and maximal eigenvalues of a symmetric matrix $A \in \mathbb{R}^{n \times n}$.

In this work we will assume that all external and internal signals in the system (1) are bounded:

Assumption 1. $\|x\|<+\infty,\|v\|<+\infty,\|u\|<+\infty$.

Although assumed to be bounded, the disturbance $v$ may have a large amplitude, and therefore special attenuation techniques have to be applied to ensure reliable estimates for the states in system (1).

The objective of this work is to design an adaptive observer for (1) under the conditions of Assumption 1. The observer has to provide estimates of the vectors $x$ and $\theta$ with an improved degree of robustness with respect to the external disturbance $v$. The proposed design procedure is completed in two steps. Firstly, an adaptive observer is designed such that the $H_{\infty}$ gain between the disturbances and the output errors is calculated (and thus it can be minimized). Secondly, an additional sliding mode output injection is applied to further reduce the influence of the disturbance components which cannot be completely rejected by the first step. The proof of stability is given for each case. An analysis of the structure of the resulting observers suggests that the augmented adaptive observer behaves better than the conventional adaptive observer with $H_{\infty}$ gain optimization. The simulation results confirm this.

An adaptive observer for the system (1) has been proposed in Zhang [2002], and takes the form:

$$
\dot{z}=A z+\phi(y, u)+G(y, u) \hat{\theta}+L(y-C z)+\Omega \dot{\hat{\theta}},
$$

where

$$
\begin{gathered}
\dot{\Omega}=(A-L C) \Omega+G(y, u), \\
\dot{\hat{\theta}}=\gamma \Omega^{T} C^{T}(y-C z) .
\end{gathered}
$$

In $(2)-(3), z \in \mathbb{R}^{n}$ is the estimate of $x, \hat{\theta} \in \mathbb{R}^{q}$ is the estimate of $\theta$, and $\Omega \in \mathbb{R}^{n \times q}$ is an auxiliary/filter variable, that helps overcome possible high relative degree obstructions in system (1). In (3) $\gamma>0$ is a design parameter, and $L$ is the observer gain that is chosen to ensure a Hurwitz property for the matrix $A-L C$. The analysis of the estimation abilities of the observer in (2), (3) is based on the errors $\delta=x-z+\Omega \tilde{\theta}$ and $\tilde{\theta}=\hat{\theta}-\theta$ whose dynamics can be shown to have the form:

$$
\begin{aligned}
& \dot{\delta}=(A-L C) \delta+B v, \\
& \dot{\tilde{\theta}}=\gamma \Omega^{T} C^{T}(C \delta-C \Omega \tilde{\theta}) .
\end{aligned}
$$

From equation (4) we conclude that $\delta \rightarrow 0$ for $v=0$ and the variable $\delta$ stays bounded for any bounded disturbance $v$. The Hurwitz property of the matrix $A-L C$ and Assumption 1 imply boundedness of the variable $\Omega$. If the signal $G(y, u)$ is persistently exciting (PE) Anderson [1977], Yuan and Wonham [1977], then due to the filtering property of the variable $\Omega$, the variable $C \Omega$ is also PE. Moreover, it is possible to show Efimov [2004] that for any bounded signal $C \delta$, the variable $\tilde{\theta}$ has a bounded response, and if $C \delta \rightarrow 0$, then $\tilde{\theta} \rightarrow 0$ also. Since as a consequence of Assumption 1 the variables $x, \delta, \tilde{\theta}$ and $\Omega$ are bounded, the variable $z$ is also bounded according to the definition of $\delta$. In addition, it is easy to verify that if $\tilde{\theta} \rightarrow 0$, then $z \rightarrow x$. Thus, we have shown that the variables $z, \Omega$ and $\hat{\theta}$ stay bounded for any bounded disturbance $v$, and for the case when $v=0$ the estimates $\hat{\theta}$ and $z$ converge to their ideal values $\theta$ and $x$ respectively. Thus the proof is based on general stability arguments, and no strict Lyapunov function has been proposed.

To improve the accuracy of estimation in the observer (2)-(3), one can design the matrix $L$ optimizing the $H_{\infty}$ gain of the system $(C, A-L C, B)$ Henry and Zolghadri [2005]. In this case the influence of the disturbance $v$ on the output $C \delta$ of the system in (4) is minimized, and implies a proportional amelioration of the error $\tilde{\theta}$ in (5). This will be proven formally in Section 3 below. Moreover, it will be shown that if $v$ is a Lipschitz function of $x$, then by proper selection of $L$ it is possible to attenuate the disturbance $v$. If the amplitude of the disturbance $v$ is large, then such an optimization could be insufficient. This problem can be addressed by modifying equation (2): this approach is later demonstrated in Section 4 using a sliding mode technique.

\section{CONVENTIONAL ADAPTIVE OBSERVER}

First let us show that the system in (5) is input-to-state stable with respect to the input $C \delta$.

Lemma 1. Let the variable $\Omega^{T} C^{T}$ be PE and bounded, i.e. $0<\rho=\|C \Omega\|<+\infty$ and there exist constants $\vartheta>0$ and $\ell>0$ such that

$$
\int_{t}^{t+\ell} \Omega(\tau)^{T} C^{T} C \Omega(\tau) d \tau \geq \vartheta I_{q} \quad \forall t \geq 0 .
$$

Then

a) there exists a continuous symmetric matrix function $P: \mathbb{R}_{+} \rightarrow \mathbb{R}^{q \times q}$ such that $\rho^{-2} I_{q} \leq 2 \gamma P(t) \leq \alpha I_{q}$ for all $t \geq 0$, where $\alpha=\gamma \eta^{-1} e^{2 \eta \ell}$ and $\eta=-0.5 \ell^{-1} \ln (1-$ $\left.\frac{\gamma \vartheta}{1+\gamma^{2} \ell^{2} \rho^{4}}\right)$;

b) for all $t \geq 0$

$\dot{P}(t)-\gamma P(t) \Omega(t)^{T} C^{T} C \Omega(t)-\gamma \Omega(t)^{T} C^{T} C \Omega(t) P(t)+I_{q}=0 ;$

c) for $S(t, \tilde{\theta})=\tilde{\theta}^{T} P(t) \tilde{\theta}$ we have for all $\tilde{\theta} \in \mathbb{R}^{q}, \delta \in \mathbb{R}^{n}$ and $t \geq 0$

$$
\dot{S} \leq-\gamma \alpha^{-1} S+0.5 \rho^{2} \alpha^{2}|C \delta|^{2} .
$$

In addition, for all $\tilde{\theta}(0) \in \mathbb{R}^{q}$ and all $t \geq 0$ the following estimate is satisfied:

$$
|\tilde{\theta}(t)| \leq \rho \sqrt{\alpha}\left[e^{-0.5 \gamma \alpha^{-1} t}|\tilde{\theta}(0)|+\rho \alpha|| C \delta||\right] .
$$

The proof is omitted for brevity. 
Remark 2. This lemma also provides an estimate on the fastest rate of decrease of the parametric estimation error $\tilde{\theta}(t)$. Indeed, the rate of decrease equals $0.5 \gamma \alpha^{-1}=$ $0.5 \eta e^{-2 \eta \ell}=g(\eta)$, where the function $g(\eta)$ for $\eta>0$ has the unique maximum value $g_{\max }=0.25 \ell^{-1} e^{-1}$ at $\eta_{\text {opt }}=0.5 \ell^{-1}$. However, as shown in the proof of Lemma $1,0<\eta \leq \eta_{\max }=0.5 \ell^{-1} \ln 2$. Since $\eta_{\text {opt }}>\eta_{\max }$ and the function $g(\eta)$ is strictly increasing for $0<\eta \leq \eta_{\text {opt }}$, then the maximal rate of error decrease that can be reached is $g\left(\eta_{\max }\right)=0.125 \ell^{-1} \ln 2$.

Note that in order to use these estimates we have to know the value of the coefficient $\rho$. The existence of such a $\rho$ follows from Assumption 1 for the Hurwitz matrix $A-L C$, but to compute it we have to know $\|x\|$, which is assumed to be unavailable.

Theorem 3. Suppose Assumption 1 is satisfied, the variable $\Omega^{T} C^{T}$ is PE, i.e. there exist constants $\vartheta>0$ and $\ell>0$ such that

$$
\int_{t}^{t+\ell} \Omega(\tau)^{T} C^{T} C \Omega(\tau) d \tau \geq \vartheta I_{q} \quad \forall t \geq 0
$$

and there exists a $n \times n$ matrix $W=W^{T}>0$ and constants $r>0, h>0$ such that

$$
\begin{gathered}
(A-L C)^{T} W+W(A-L C)+0.5 r \alpha^{2} C^{T} C \\
+h W B B^{T} W+\gamma \alpha^{-1} W \leq 0
\end{gathered}
$$

where $\alpha=\gamma \eta^{-1} e^{2 \eta \ell}, \eta=-0.5 \ell^{-1} \ln \left(1-\frac{\gamma \vartheta}{1+\gamma^{2} \ell^{2}\|C \Omega\|^{4}}\right)$ and $\gamma>0$. Then in system (1), (2), (3) all solutions are bounded for all $t \geq 0$, and

$$
\begin{aligned}
{\left[\begin{array}{c}
|x(t)-z(t)| \\
|\tilde{\theta}(t)|
\end{array}\right] \leq } & (1+\| \Omega||)\left\{\sqrt{\frac{\alpha_{2}}{\alpha_{1}}} e^{0.5 \gamma \alpha^{-1} t}\left|\left[\begin{array}{c}
\delta(0) \\
\tilde{\theta}(0)
\end{array}\right]\right|\right. \\
& \left.+\sqrt{\frac{\alpha}{\alpha_{1} \gamma h}}\|v\|\right\}
\end{aligned}
$$

where $\alpha_{1}=\min \left\{\lambda_{\min }(W), 0.5 r \gamma^{-1}\|C \Omega\|^{-4}\right\}, \quad \alpha_{2}=$ $\max \left\{\lambda_{\max }(W), 0.5 r\|C \Omega\|^{-2} \gamma^{-1} \alpha\right\}$.

Proof. It follows from Assumption 1 the variable $\Omega$ is bounded for any Hurwitz matrix $A-L C$ (the stability of this matrix follows from the Riccati equation for the matrix $W$ ), and it is also a continuous matrix function of time. Define $\rho=\|C \Omega\|<+\infty$. Consider as a candidate Lyapunov function $V(\delta)=\delta^{T} W \delta$, then

$$
\dot{V}=\delta^{T}\left[(A-L C)^{T} W+W(A-L C)\right] \delta+2 \delta^{T} W B v .
$$

Note that $2 \delta^{T} W B v \leq h \delta^{T} W B B^{T} W \delta+h^{-1} v^{T} v$, then

$$
\begin{aligned}
\dot{V} \leq & \delta^{T}\left[(A-L C)^{T} W+W(A-L C)\right. \\
& \left.+h W B B^{T} W\right] \delta+h^{-1}|v|^{2} .
\end{aligned}
$$

Define $U(t, \delta, \tilde{\theta})=V(\delta)+r \rho^{-2} S(t, \tilde{\theta})$, then using the result of Lemma 1 we have

$$
\begin{aligned}
\dot{U} \leq & \delta^{T}\left[(A-L C)^{T} W+W(A-L C)+h W B B^{T} W\right] \delta \\
& -\gamma r \rho^{-2} \alpha^{-1} S+0.5 r \alpha^{2}|C \delta|^{2}+h^{-1}|v|^{2} \\
\leq & \delta^{T}\left[(A-L C)^{T} W+W(A-L C)+0.5 r \alpha^{2} C^{T} C\right. \\
& \left.+h W B B^{T} W\right] \delta-\gamma r \rho^{-2} \alpha^{-1} S+h^{-1}|v|^{2} \\
\leq & \delta^{T}\left[(A-L C)^{T} W+W(A-L C)+0.5 r \alpha^{2} C^{T} C\right. \\
& \left.+h W B B^{T} W+\gamma \alpha^{-1} W\right] \delta-\gamma \alpha^{-1} U+h^{-1}|v|^{2} \\
\leq & -\gamma \alpha^{-1} U+h^{-1}|v|^{2} .
\end{aligned}
$$

Note that according to the result of Lemma 1 the following inequalities hold:

$$
\begin{gathered}
\lambda_{\min }(W)|\delta|^{2}+0.5 r \gamma^{-1} \rho^{-4}|\tilde{\theta}|^{2} \leq U(t, \delta, \tilde{\theta}) \\
\leq \lambda_{\max }(W)|\delta|^{2}+0.5 r \rho^{-2} \gamma^{-1} \alpha|\tilde{\theta}|^{2}
\end{gathered}
$$

Therefore, for all $\delta(0) \in \mathbb{R}^{n}$ and $\tilde{\theta}(0) \in \mathbb{R}^{q}$, and for all $t \geq 0$ we obtain:

$$
\left|\left[\begin{array}{l}
\delta(t) \\
\tilde{\theta}(t)
\end{array}\right]\right| \leq \sqrt{\frac{\alpha_{2}}{\alpha_{1}}} e^{0.5 \gamma \alpha^{-1} t}\left|\left[\begin{array}{l}
\delta(0) \\
\tilde{\theta}(0)
\end{array}\right]\right|+\sqrt{\frac{\alpha}{\alpha_{1} \gamma h}}\|v\| .
$$

Since the variables $x, \delta, \Omega$ and $\tilde{\theta}$ are bounded, then the variable $z$ has the same property $(z=x-\delta+\Omega \tilde{\theta})$, and in addition

$$
\begin{aligned}
|x(t)-z(t)| & \leq|\delta(t)|+|| \Omega|||\tilde{\theta}(t)| \\
& \leq(1+\| \Omega||)\left|\left[\begin{array}{c}
\delta(t) \\
\tilde{\theta}(t)
\end{array}\right]\right|,
\end{aligned}
$$

which gives the required estimate.

This theorem estimates the $H_{\infty}$ gain of the observer from the input $v$ to the estimation errors $\tilde{\theta}$ and $x-z$. A minor modification of the LMI for $W$ in Theorem 3 is needed in order to ensure the $H_{\infty}$ gain from $v$ to a generic output $Z(x-z)$, where $Z$ is a matrix (this modification is omitted). Next, a minimization of the $H_{\infty}$ gain can be performed, using the nonlinear optimization routines (the detailed presentation of this part is skipped for brevity).

The Riccati equation (6) depends on $\alpha$ which is a function of the PE parameters $\ell, \vartheta$ and $\rho$, which can be unknown a priori. However, this equation can be rewritten as

$$
\begin{gathered}
(A-L C)^{T} W+W(A-L C)+r_{1} C^{T} C \\
+h W B B^{T} W+r_{2} W \leq 0
\end{gathered}
$$

where $r_{1}=0.5 r \alpha^{2}$ and $r_{2}=\gamma \alpha^{-1}$ are the new tuning parameters. If this Riccati inequality is satisfied for some $h>0, r_{1}>0$ and $r_{2}>0$, and the PE property is true for $\Omega^{T} C^{T}$, then there exist $\gamma>0$ and $r>0$ such that (6) is satisfied.

Remark 4. It is straightforward to verify that if the disturbance $v$ contains a globally Lipschitz nonlinear function of the state, then augmenting the value of $h$, and introducing a minor modification in (2), it is possible to attenuate this type of uncertainty. Indeed, let $v(t)=\mu(x)+\tilde{v}(t)$, where $\mu: \mathbb{R} \rightarrow \mathbb{R}^{s}$ is a function which for any $x, x^{\prime} \in \mathbb{R}^{n}$ satisfies the inequality $\left|\mu(x)-\mu\left(x^{\prime}\right)\right| \leq L_{\mu}\left|x-x^{\prime}\right|$ for some $L_{\mu}>0$ and $\tilde{v}: \mathbb{R}_{+} \rightarrow \mathbb{R}^{s}$ is a new bounded exogenous input. Then introducing in (2) an additional term $B \mu(z)$ and solving the LMI of Theorem 3 for a sufficiently large value of $h>0$, complete compensation of the influence of $\mu(x)$ can be achieved.

\section{SLIDING-MODE ADAPTIVE OBSERVER}

In this case the observer equation (2) is modified as follows:

$$
\begin{gathered}
\dot{z}=A z+\phi(y, u)+G(y, u) \hat{\theta}+L(y-C z)+\Omega \dot{\hat{\theta}} \\
+k B \operatorname{sign}[F(y-C z)],
\end{gathered}
$$

where $k>0$ is a design constant, $F \in \mathbb{R}^{n \times p}$ is a matrix to be designed and $\operatorname{sign}[v]=v /|v|$ for a vector $v \in \mathbb{R}^{m}$.

Theorem 5. Let assumption 1 be satisfied and let $k=\|v\|$. Assume the variable $\Omega^{T} C^{T}$ is PE, i.e. there exist $\vartheta>0$ and $\ell>0$ such that 


$$
\int_{t}^{t+\ell} \Omega(\tau)^{T} C^{T} C \Omega(\tau) d \tau \geq \vartheta I_{q} \quad \forall t \geq 0,
$$

and suppose there exists a matrix $W=W^{T}>0$, a matrix $F \in \mathbb{R}^{n \times p}$ and constants $r>0, \omega>0$ such that

$$
\begin{gathered}
(A-L C)^{T} W+W(A-L C)+\gamma \alpha^{-1} W \\
+\left(0.5 r \alpha^{2}+\omega\right) C^{T} C \leq 0 \\
W B=C^{T} F^{T}
\end{gathered}
$$

where

$$
\begin{gathered}
\alpha=\gamma \eta^{-1} e^{2 \eta \ell}, \gamma>0 \\
\eta=-0.5 \ell^{-1} \ln \left(1-\frac{\gamma \vartheta}{1+\gamma^{2} \ell^{2}\|C \Omega\|^{4}}\right) .
\end{gathered}
$$

Then in the system (1), (3), (7), all variables $z, \Omega$ and $\tilde{\theta}$ are bounded and

$$
\begin{aligned}
{\left[\begin{array}{c}
|x(t)-z(t)| \\
|\tilde{\theta}(t)|
\end{array}\right] \leq } & (1+\| \Omega||)\left(\sqrt{\frac{\alpha_{2}}{\alpha_{1}}} e^{-0.5 \gamma \alpha^{-1} \kappa t}\left|\left[\begin{array}{c}
\delta(0) \\
\tilde{\theta}(0)
\end{array}\right]\right|\right. \\
& \left.+2 \sqrt{\frac{\alpha}{\alpha_{1} \omega \gamma \kappa}}|F|\|v\|\right) .
\end{aligned}
$$

where $\kappa=\frac{0.5 r}{0.5 r+\omega\|C \Omega\|^{2}}$,

$$
\begin{gathered}
\alpha_{1}=\min \left\{\lambda_{\min }(W), \gamma^{-1}\left(0.5 r\|C \Omega\|^{-4}+\omega\right)\right\}, \\
\alpha_{2}=\max \left\{\lambda_{\max }(W), \gamma^{-1}\left(0.5 r\|C \Omega\|^{-2}+\omega\right) \alpha\right\} .
\end{gathered}
$$

Proof. As in the previous theorem the variable $\Omega$ is bounded for any Hurwitz matrix $A-L C$ (the stability of this matrix follows from the Lyapunov equation for the matrix $W$ ) and let $\rho=\|C \Omega\|$. The error $\delta=x-z+\Omega \tilde{\theta}$ for the system $(1),(3),(7)$ has the following dynamics:

$$
\dot{\delta}=(A-L C) \delta+B\{v-k \operatorname{sign}[F(y-C z)]\} .
$$

Consider three Lyapunov function components given by $V(\delta)=\delta^{T} W \delta, Z(\tilde{\theta})=\gamma^{-1} \tilde{\theta}^{T} \tilde{\theta}$ and $S(t, \tilde{\theta})=\tilde{\theta}^{T} S(t) \tilde{\theta}$. These have the property that

$$
\begin{aligned}
\dot{V}= & \delta^{T}\left[(A-L C)^{T} W+W(A-L C)\right] \delta+ \\
& +2 \delta^{T} C^{T} F^{T}\{v-k \operatorname{sign}[F(y-C z)]\} \\
\leq & -\delta^{T}\left[\gamma \alpha^{-1} W+\left(0.5 r \alpha^{2}+\omega\right) C^{T} C\right] \delta \\
& +2[y-C z]^{T} F^{T}\{v-k \operatorname{sign}[F(y-C z)]\} \\
& +2 \tilde{\theta}^{T} \Omega^{T} C^{T} F^{T}\{v-k \operatorname{sign}[F(y-C z)]\} \\
\leq & -\delta^{T}\left[\gamma \alpha^{-1} W+\left(0.5 r \alpha^{2}+\omega\right) C^{T} C\right] \delta \\
& +4\left|\tilde{\theta}^{T} \Omega^{T} C^{T}\right||F||v| \mid \\
\dot{Z}= & 2 \tilde{\theta}^{T} \Omega^{T} C^{T}(C \delta-C \Omega \tilde{\theta}) \\
\leq & |C \delta|^{2}-\left|\tilde{\theta}^{T} \Omega^{T} C^{T}\right|^{2} \\
\dot{S} \leq & -0.5 \tilde{\theta}^{T} \tilde{\theta}+0.5 \rho^{2} \alpha^{2}|C \delta|^{2} .
\end{aligned}
$$

Introduce a new candidate Lyapunov function given by $U(t, \delta, \tilde{\theta})=V(\delta)+\omega Z(\tilde{\theta})+r \rho^{-2} S(t, \tilde{\theta})$, then we obtain

$$
\begin{aligned}
\dot{U} \leq & -\gamma \alpha^{-1} \delta^{T} W \delta-0.5 r \rho^{-2} \tilde{\theta}^{T} \tilde{\theta} \\
& +4\left|\tilde{\theta}^{T} \Omega^{T} C^{T}\right||F|\|v\|-\omega\left|\tilde{\theta}^{T} \Omega^{T} C^{T}\right|^{2} \\
\leq & -\gamma \alpha^{-1} \delta^{T} W \delta-0.5 r \rho^{-2} \tilde{\theta}^{T} \tilde{\theta}+4 \omega^{-1}|F|^{2}|| v \|^{2},
\end{aligned}
$$

where the inequality $4 s|F|\left|v\left\|-\omega s^{2} \leq 4|F|^{2}\right\| v \|^{2} / \omega\right.$, which is satisfied for all $s \in \mathbb{R}_{+}$, has been used in the last step of transformations. Since

$$
\begin{aligned}
\gamma^{-1}\left(0.5 r \rho^{-4}+\omega\right)|\tilde{\theta}|^{2} & \leq \omega Z(\tilde{\theta})+r \rho^{-2} S(t, \tilde{\theta}) \\
& \leq \gamma^{-1}\left(0.5 r \rho^{-2}+\omega\right) \alpha|\tilde{\theta}|^{2}
\end{aligned}
$$

we finally obtain

$$
\dot{U} \leq-\gamma \alpha^{-1} \kappa U+4 \omega^{-1}|F|^{2}\|v\|^{2}
$$

and for all $t \geq 0$

$$
\begin{aligned}
\left|\left[\begin{array}{c}
\delta(t) \\
\tilde{\theta}(t)
\end{array}\right]\right| \leq & \sqrt{\frac{\alpha_{2}}{\alpha_{1}}} e^{-0.5 \gamma \alpha^{-1} \kappa t}\left|\left[\begin{array}{c}
\delta(0) \\
\tilde{\theta}(0)
\end{array}\right]\right| \\
& +2 \sqrt{\frac{\alpha}{\alpha_{1} \omega \gamma \kappa}}|F|\|v\| .
\end{aligned}
$$

According to the proof above, the variables $x, \delta, \Omega$ and $\tilde{\theta}$ are bounded, consequently the variable $z$ has the same property $(z=x-\delta+\Omega \tilde{\theta})$, and again

$$
|x(t)-z(t)| \leq(1+\|\Omega\|)\left|\left[\begin{array}{c}
\delta(t) \\
\tilde{\theta}(t)
\end{array}\right]\right|,
$$

which gives the required estimate.

Remark 6. Comparing with the case of Theorem 3, in Theorem 5, the accuracy of estimation is improved, since in part, the disturbance $v$ is compensated by $\operatorname{sign}(\cdot)$. Indeed, the dynamics of the state estimation error $e=x-z$ yields the following differential equation

$$
\begin{aligned}
\dot{e}= & \left(A-\left[L+\gamma \Omega \Omega^{T} C^{T}\right] C\right) e-G(y, u) \tilde{\theta} \\
& +B[v-k \operatorname{sign}(F C e)],
\end{aligned}
$$

which for $k=0$ reduces to (2). Consider the Lyapunov function $V(e)=e^{T} W e$, then

$$
\begin{aligned}
\dot{V} \leq & e^{T}\left\{(A-L C)^{T} W+W(A-L C)\right\} e \\
& +2 e^{T} C^{T} F^{T}(v-k \operatorname{sign}(F C e)) \\
& -2 e^{T} W\left[\gamma \Omega \Omega^{T} C^{T} C e+G(y, u) \tilde{\theta}\right] \\
\leq & e^{T}\left\{(A-L C)^{T} W+W(A-L C)\right\} e \\
& -2|F C e|(k-\|v\|) \\
& -2 e^{T} W\left[\gamma \Omega \Omega^{T} C^{T} C e+G(y, u) \tilde{\theta}\right] .
\end{aligned}
$$

Therefore, for the observer in (7), with $k=\|v\|$, the term proportional to $|F C e|$ disappears and the influence of $v$ is cancelled. The Lyapunov equation for the matrix $W$ is also simpler than in the case of Theorem 5 (and an additional decision variable $F$ appears).

In order to find a solution to the LMI in (8) we have to reformulate it, to avoid direct dependence on $\alpha$. To this end it can be rewritten as follows:

$$
(A-L C)^{T} W+W(A-L C)+r_{1} C^{T} C+r_{2} W \leq 0,
$$

where $r_{1}=\left(0.5 r \alpha^{2}+\omega\right)$ and $r_{2}=\gamma \alpha^{-1}$ are new tuning parameters.

Remark 7. The introduction of the "structural constraint" $W B=C^{T} F^{T}$ in (8) imposes limitation on the case of triple $(A, B, C)$ to which these results are applicable. However a constructive approach to solving such problems is given in Edwards et al. [2007]. Equivalent conditions can be found in Lemma 1 of Efimov and Fradkov [2006]. 


\section{EXAMPLE}

Consider a nonlinear pendulum:

$$
\begin{gathered}
n=2, A=\left[\begin{array}{cc}
0 & 1 \\
0 & -\beta
\end{array}\right], B=\left[\begin{array}{l}
1 \\
0
\end{array}\right], C=\left[\begin{array}{cc}
1 & 0
\end{array}\right], \\
0 \\
\phi(y, u)=\left[\begin{array}{c}
0 \\
u-\varpi^{2} \sin (y) \\
v(t)=\sin (2 t) .
\end{array}\right], G(y, u)=\left[\begin{array}{c}
0 \\
\sin (y)
\end{array}\right],
\end{gathered}
$$

The values of the parameters are $\varpi=1, \beta=0.1, \theta=0.5$. Let $L=[10,0]^{T}$, then if $P=\operatorname{diag}[1,10]$ all the conditions of Theorem 3 hold for $h=10, r_{1}=40$ and $r_{2}=0.2$. The conditions of Theorem 5 are satisfied for $F=1, r_{1}=50$ and $r_{2}=0.2$. In both cases the simulations have been performed for $\gamma=20$.

The results of the numerical experiments are presented in figures 1 and 2 . The parameter estimates are shown in Fig. 1, and the variable $e_{2}=x_{2}-z_{2}$ is plotted in Fig. 2 . The trajectories of the conventional observer (2),(3) are denoted by solid lines (red), whilst the trajectories of the sliding-mode observer (3),(7) are denoted by dashed lines (blue). It can be seen from these results the sliding mode observer $(3),(7)$ ensures better attenuation of disturbances.

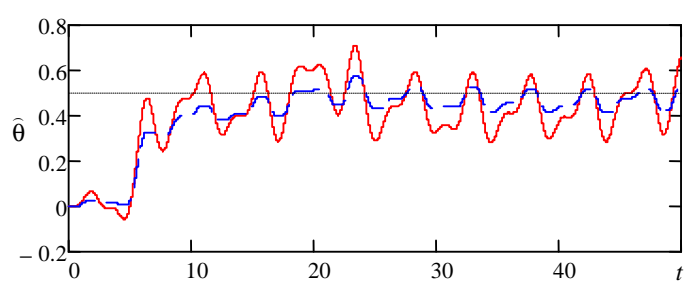

Fig. 1. The estimates of unknown parameter

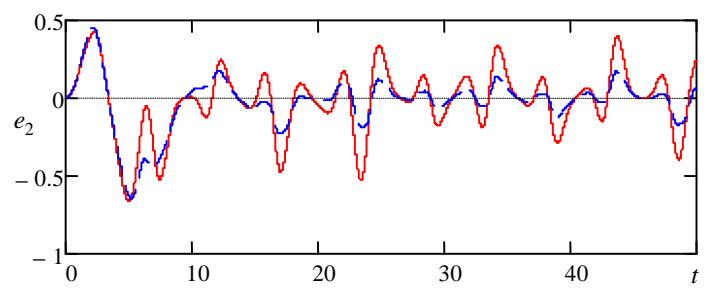

Fig. 2. The unmeasured state component estimation error

\section{CONCLUSIONS}

The problem studied in this paper is that of improving the performance of the adaptive observer originally proposed in Zhang [2002]. Following Yan and Edwards [2008], a scheme augmented by including a sliding mode term is proposed. First, the $H_{\infty}$ gain with respect to external disturbances is evaluated to minimize the effect of disturbances on the output errors in a $H_{\infty}$ sense. Next, a slidingmode modification of the adaptive observer equations from Zhang [2002] is proposed in order to improve disturbance attenuation. The conditions for stability of the new scheme have been established. Numerical simulations confirm the accuracy improvement obtained from the newly proposed observer with respect to that proposed in Zhang [2002].

\section{REFERENCES}

B.D.O. Anderson. Exponential stability of linear equations arising in adaptive identification. IEEE Trans. Automat. Control, 22:83-88, 1977.

G. Besanon, editor. Nonlinear observers and applications, volume 363 of Lecture Notes in Control and Inforamtion Science. Springer Verlag, Berlin, 2007.

C. Edwards, Xing-Gang Yan, and S.K. Spurgeon. On the solvability of the constrained lyapunov problem. Automatic Control, IEEE Transactions on, 52(10):19821987, 2007. ISSN 0018-9286.

Denis Efimov and Alexander Fradkov. Adaptive tuning to bifurcation for time-varying nonlinear systems. Automatica, 42:417-425, 2006.

D.V. Efimov. Robust adaptive nonlinear partial observers for time-varying chaotic systems. In Proc. IEEE CDC, Atlantis, Paradise Island, Bahamas, December 2004. IEEE.

A.L. Fradkov, H. Nijmeijer, and A.Yu. Markov. Adaptive observer-based synchronization for communications. Int. J. Bifurcat. Chaos, 10(12):2807-2814, 2000.

A.L. Fradkov, V.O. Nikiforov, and B.R. Andrievsky. Adaptive observers for nonlinear nonpassifiable systems with application to signal transmission. In Proc. 41th IEEE Conf. Decision and Control, pages 4706-4711, Las Vegas, Dec. 2002. IEEE.

D. Henry and A. Zolghadri. Design of fault diagnosis filters: a multi-objective approach. Journal of the Franklin Institute, 342:421-446, 2005.

Y. Shtessel, C. Edwards, and L. Fridman. Sliding Mode Control and Observation. Birkhauser, 2013.

A. $\mathrm{Xu}$ and $\mathrm{Q}$. Zhang. Residual generation for fault diagnosis in linear time-varying systems. IEEE Trans. Autom. Control, 49(5):767-772, 2004.

X.G. Yan and C. Edwards. Fault estimation for single output nonlinear systems using an adaptive sliding mode observer. IET Control Theory and Applications, 2:841-850, 2008.

J. S.-C. Yuan and W.M. Wonham. Probing signals for model reference identification. IEEE Trans. Automat. Control, 22:530-538, 1977.

Q. Zhang. Adaptive observer for multiple-input-multipleoutput (mimo) linear time varying systems. IEEE Trans. Autom. Control, 47(3):525-529, 2002. 